\title{
ATRIBUTOS QUÍMICOS E MICROBIANOS DE SOLOS SOB DIFERENTES COBERTURAS VEGETAIS NO NORTE DO ESTADO DO RIO DE JANEIRO(1)
}

\author{
Emanuela Forestieri da Gama-Rodrigues ${ }^{(2)}$, Antonio Carlos da Gama- \\ Rodrigues $^{(2,5)}$, Gleicia Miranda Paulino ${ }^{(3)}$ \& Avilio Antonio Franco ${ }^{(4,5)}$
}

\section{RESUMO}

Os objetivos do presente estudo foram avaliar a influência de Acacia auriculiformis (acácia), Mimosa caesalpiniifolia (sabiá) e Corymbia citriodora (eucalipto), comparadas às coberturas de capoeira e pastagem, sobre os atributos químicos e microbianos do solo. As espécies apresentaram sobrevivência no campo acima de 70 \% e bom desenvolvimento em altura e diâmetro (DAP). Entre as leguminosas, o sabiá apresentou a maior taxa de sobrevivência, enquanto a acácia mostrou melhor desenvolvimento, especialmente entre os 27 e 44 meses. As espécies florestais, na fase inicial de desenvolvimento, revelaram-se promissoras em melhorar a fertilidade do solo, apesar da redução dos teores de matéria orgânica. A pastagem mostrou potencial de manter o teor de MO e melhorar a fertilidade do solo. $\mathrm{O} \mathrm{C} \mathrm{e} \mathrm{N}$ microbiano foram mais discriminantes que $\mathrm{o} \mathrm{C}$ orgânico do solo às mudanças na cobertura vegetal. Os índices microbianos $\left(q \mathrm{CO}_{2}\right.$ - quociente metabólico, CBM/C - relação C da biomassa microbiana/C orgânico, NBM/N - relação $\mathrm{N}$ da biomassa microbiana/N total e relação $\mathrm{C} / \mathrm{N}$ microbiana) foram mais discriminantes que os atributos químicos (C orgânico e $\mathrm{N}$ total) e microbianos (CBM - carbono da biomassa microbiana, NBM - N da biomassa microbiana e RA respiração acumulada) em aferir a dissimilaridade entre as coberturas vegetais.

Termos de indexação: qualidade de solo, fertilidade do solo, espécies florestais, biomassa microbiana, atividade microbiana, solos degradados.

\footnotetext{
(1) Parte da Tese de Mestrado do terceiro autor apresentada ao Programa de Pós-Graduação em Produção Vegetal, Universidade Estadual do Norte Fluminense - UENF. Pesquisa parcialmente financiada com recursos da FAPERJ e da Embrapa/PRODETAB. Recebido para publicação em agosto de 2007 e aprovado em junho de 2008.

(2) Professor Associado do Laboratório de Solos do Centro de Ciências e Tecnologias Agropecuárias - CCTA, Universidade Estadual do Norte Fluminense - UENF. CEP 28013-602 Campos dos Goytacazes (RJ). E-mail: emanuela@uenf.br; tonygama@uenf.br

(3) Doutoranda do Programa de Pós-Graduação em Produção Vegetal, UENF.

(4) Pesquisador da Embrapa Agrobiologia. Rod. BR 465, km 47, CEP 23851-970 Seropédica (RJ). E-mail: avilio@cnpab.embrapa.br

(5) Bolsista FAPERJ.
} 


\title{
SUMMARY: CHEMICAL AND MICROBIAL ATTRIBUTES OF SOILS UNDER DIFFERENT SOIL COVERS IN NORTHERN RIO DE JANEIRO STATE, BRAZIL
}

\begin{abstract}
The objectives of this study were to evaluate the influence of three soil cover species Acacia auriculiformis, Mimosa caesalpiniifolia and Corymbia citriodora compared to pasture and natural secondary forest on soil chemical and microbial attributes. The field survival rate of the species reached over $70 \%$ and the development in height and diameter (DAP) was adequate. The survival rate of Mimosa caesalpiniifolia was the highest among the legume species, while the development of Acacia auriculiformis was the best, mainly between 27 and 44 months. The forest species improved soil fertility adequately in the initial development stages, in spite of the reduction in the levels of organic matter. The potential of pasture to maintain the level of organic matter and improve soil fertility was shown. Microbial $C$ and $N$ were more discriminating, regarding changes in land cover than soil $C$ content. The microbial quotients (q $\mathrm{CO}_{2}, C B M / C, N B M / N$ and microbial $C / N$ ) detected differences among land covers more clearly than the chemical ( $C$ and $N$ content) and microbial (CBM, NBM and RA) attributes.
\end{abstract}

Index terms: soil quality, soil fertility, forest species, microbial biomass, microbial activity, degraded soils.

\section{INTRODUÇÃO}

Na região Norte Fluminense, a cobertura atual de Mata Atlântica é inferior a $5 \%$ da mata original, devido ao longo período de atividades agropecuárias, especialmente da cana-de-açúcar e do café, com uso regular do fogo e mecanização intensiva. Isso resultou não apenas na drástica redução do maciço florestal, como também num elevado grau de degradação dos solos. A conseqüência disso foi a decadência socioeconômica da região, na qual as atividades de uso atual das terras são pastagens também degradadas (Gama-Rodrigues \& May, 2001). Em face dessa realidade, técnicas e estratégias de recuperação de terras degradadas têm sido desenvolvidas e empregadas no sentido de favorecer os mecanismos naturais que permitem a reação do ecossistema aos diferentes graus de perturbação (Campello, 1998).

Nesse sentido, o uso da cobertura vegetal com espécies arbóreas, sobretudo leguminosas associadas às bactérias fixadoras de $\mathrm{N}_{2}$ e fungos micorrízicos, constitui uma técnica que tem mostrado viabilidade para recuperação e sustentabiliade dos solos das regiões tropicais, pela influência positiva em vários atributos de qualidade do solo (Franco, 1996). As principais influências da cobertura florestal na qualidade dos solos, mediante a reciclagem de nutrientes, são o aumento do teor de $\mathrm{MO}$ e da disponibilidade de nutrientes, a melhoria das propriedades físicas e a otimização da atividade biológica (Young, 1989; Franco \& Faria, 1997; Gama-Rodrigues et al., 1999). O plantio de árvores, além de contribuir para a manutenção ou melhoria dos processos ecológicos, também reduziria a pressão de exploração sobre os remanescentes de floresta natural e promoveria uma alternativa socioeconômica de desenvolvimento regional (Láng, 1994).
A estimativa de atributos edáficos relacionados à sua funcionalidade possibilitaria monitorar indiretamente a qualidade do solo (Reinert, 1998) e sua utilização como indicadores importantes para o planejamento e a avaliação de práticas de manejo que sejam ecológica e economicamente viáveis (Doran \& Zeiss, 2000; Arshad \& Martin, 2002; Bouma, 2002). Contudo, no Brasil, a utilização desses indicadores em áreas degradadas é recente, e as pesquisas relacionadas à recuperação dessas áreas estão ainda formando suas bases de dados, por meio de programas de monitoramento das atividades empregadas no sentido de melhorar a qualidade do ambiente (Campello et al., 2000).

A biomassa microbiana tem sido sugerida como indicador biológico da qualidade da MO do solo para aferição da sustentabilidade de sistemas de produção (Mele \& Carter,1993). Por constituir a maior parte da fração ativa da MO, a biomassa microbiana é, conseqüentemente, mais sensível que o $\mathrm{C}$ orgânico e $\mathrm{N}$ total em aferir mudanças nos teores e na qualidade da MO do solo decorrentes de manejo e práticas de cultivo (Gama-Rodrigues, 1999).

Os objetivos deste estudo foram avaliar a influência de Acacia auriculiformis (acácia), Mimosa caesalpiniifolia (sabiá) e Corymbia citriodora (eucalipto), comparadas às coberturas de capoeira e pastagem, sobre os atributos químicos e microbianos do solo.

\section{MATERIAL E MÉTODOS}

O trabalho foi realizado na Fazenda Carrapeta, no município de Conceição de Macabu, RJ (21 ${ }^{\circ} 37^{\prime}$ 'S e $\left.42^{\circ} 05^{\prime} \mathrm{W}\right)$. O clima da região, pela classificação de 
Köppen, é do tipo Am, quente e úmido. De acordo com informações locais, a temperatura está em torno de $26^{\circ} \mathrm{C}$ e a precipitação pluvial média anual é de $1.400 \mathrm{~mm}$, com período chuvoso entre outubro e março e seco entre junho e setembro. O solo é um Latossolo Vermelho-Amarelo de textura argilo-franco-arenosa, em todas as coberturas vegetais. O relevo é ondulado, com declividade em torno de $35 \mathrm{~cm} \mathrm{~m}^{-1}$.

A área experimental constituiu-se de cinco coberturas vegetais em parcelas de $1.500 \mathrm{~m}^{2}$ $(75 \times 20 \mathrm{~m})$. As parcelas experimentais de cada cobertura foram dispostas adjacentes uma à outra, na mesma cota de altitude. As coberturas vegetais foram constituídas de plantios puros das espécies arbóreas Acacia auriculiformis (acácia) e Mimosa caesalpiniifolia (sabiá), inoculadas com estirpes selecionadas de bactérias fixadoras de $\mathrm{N}_{2}$ atmosférico (BR 3465 e BR 3609 para Acacia auriculiformis e BR 3407 e 3446 para Mimosa caesalpiniifolia) e fungos micorrízicos (um combinado de Gigaspora margarita e Glomus clarum para todas as espécies de leguminosas) e o eucalipto. As outras duas coberturas vegetais, adjacentes aos plantios puros, utilizados como referência, ambas com aproximadamente 40 anos de idade, foram um pasto degradado, que representa a vegetação anterior ao plantio das espécies arbóreas, com predomínio de capim-gordura (Melinis minutiflora), grama-pernambuco (Paspalum maritimum) e sapê (Imperata brasiliensis); e um fragmento florestal de Mata Atlântica em sucessão secundária, com espécies em diferentes estádios sucessionais (capoeira). A escolha da capoeira como um dos ecossistemas de referência é uma das estratégias utilizadas para avaliar alterações do solo em decorrência do tipo de uso e de técnicas de manejo por meio da comparação de características do solo manejado com as do solo não-manejado, sob vegetação natural (Barros \& Comerford, 2002).

O plantio das espécies arbóreas foi realizado em dezembro de $1998 \mathrm{com}$ mudas de $30 \mathrm{~cm}$ de altura e, no caso das leguminosas, com ótima nodulação e micorrização (bom crescimento das mudas e presença de nódulos ativos). O espaçamento utilizado foi de $3 \times 2 \mathrm{~m}$, em covas de $20 \times 20 \times 20 \mathrm{~cm}$, às quais foram adicionados $150 \mathrm{~g}$ de superfosfato simples e $10 \mathrm{~g}$ de cloreto de potássio. Nas covas de eucalipto foram adicionados também $10 \mathrm{~g}$ de uréia. O nível de sobrevivência das espécies florestais foi avaliado aos 15 meses de plantio, sendo o crescimento avaliado aos 15, 27 e 44 meses de idade, por meio de medição da altura total e do diâmetro à altura do peito (DAP) das plantas.

Aos 32 meses de idade, foram coletadas, em cada cobertura vegetal, quatro amostras compostas de solo, sendo cada uma constituída de 10 amostras simples ao acaso, nas entrelinhas de plantio, na profundidade de $0-10 \mathrm{~cm}$. Foram feitas as seguintes determinações, conforme os métodos descritos em Embrapa (1997): $\mathrm{pH}\left(\mathrm{H}_{2} \mathrm{O}\right)$; $\mathrm{P}$ e $\mathrm{K}$ extraíveis, por Mehlich-1 e determinados, respectivamente, por colorimetria e fotometria de chama; $\mathrm{Ca}, \mathrm{Mg}$ e $\mathrm{Al}$ trocáveis, por $\mathrm{KCl}$ $1 \mathrm{~mol} \mathrm{~L}^{-1}$, sendo $\mathrm{Ca}$ e $\mathrm{Mg}$ determinados por espectrofotometria de absorção atômica e o $\mathrm{Al}$ por titulação com $\mathrm{NaOH} 0,025 \mathrm{~mol} \mathrm{~L}^{-1}$; e $\mathrm{H}+\mathrm{Al}$, por acetato de cálcio $0,5 \mathrm{~mol} \mathrm{~L}^{-1} \mathrm{a} \mathrm{pH} \mathrm{7,0.} \mathrm{O} \mathrm{N}$ total foi determinado pelo método Kjeldahl, e o C orgânico, por oxidação com $\mathrm{K}_{2} \mathrm{Cr}_{2} \mathrm{O}_{7} 1,25 \mathrm{~mol} \mathrm{~L}^{-1} \mathrm{em}$ meio ácido (Anderson \& Ingram, 1993).

Para determinação do $\mathrm{C}$ e $\mathrm{N}$ da biomassa microbiana do solo, foram coletadas quatro amostras compostas, cada uma constituída de 10 amostras simples, por cobertura vegetal, aos 39 meses de idade. A amostragem foi realizada ao acaso, nas entrelinhas de plantio e na profundidade de $0-10 \mathrm{~cm}$. As amostras de solo foram destorroadas, passadas em peneira de $2 \mathrm{~mm}$ de malha e homogeneizadas, retirando-se as raízes e os resíduos visíveis de plantas e animais do solo. Para eliminar o efeito do peneiramento, antes das análises microbianas, as amostras de solo foram incubadas em recipientes fechados, em temperatura entre 22 e $25^{\circ} \mathrm{C}$, no escuro, por sete dias, contendo um frasco com água e outro com $\mathrm{NaOH} 1 \mathrm{~mol} \mathrm{~L}^{-1}$, para absorver o $\mathrm{CO}_{2}$ liberado do solo. Após essa préincubação, padronizou-se a umidade para $40 \%$ da capacidade máxima de saturação do solo (Grisi, 1995).

O método da fumigação-extração foi utilizado para estimar o C (Vance et al., 1987) e o N (Joergensen \& Brookes, 1990) da biomassa microbiana, respectivamente CBM e NBM. De cada amostra, foram pesadas seis subamostras de $20 \mathrm{~g}$ de solo (três fumigadas e três não-fumigadas); as amostras foram fumigadas com clorofórmio isento de álcool por $24 \mathrm{~h}$. A extração foi feita com $\mathrm{K}_{2} \mathrm{SO}_{4} 0,5 \mathrm{~mol} \mathrm{~L}^{-1}$ a pH entre 6,5 e 6,8 . A quantificação do $\mathrm{C}$ foi realizada por oxidação do $\mathrm{C}$ por permanganato de K (Bartlett \& Ross, 1988), e a do $\mathrm{N}$, pela determinação do N-reativo de ninidrina (Joergensen \& Brookes, 1990).

A atividade microbiana foi medida em termos de respiração do solo, estimando-se a quantidade de C$\mathrm{CO}_{2}$ liberado em sete dias de incubação. Pesaram-se, de cada amostra composta, três subamostras de $50 \mathrm{~g}$ de solo, e cada uma foi colocada em pote contendo um frasco com $10 \mathrm{~mL}$ de $\mathrm{NaOH} 1 \mathrm{~mol} \mathrm{~L}^{-1}$, mantido hermeticamente fechado. A respiração do solo foi obtida por meio da titulação do $\mathrm{NaOH}$ com $\mathrm{HCl}$ $0,5 \mathrm{~mol} \mathrm{~L}^{-1}$.

Determinou-se a relação $\mathrm{C} / \mathrm{N}$ microbiana do solo, e o $q \mathrm{CO}_{2}$ foi calculado pela relação entre a respiração basal e o C da biomassa microbiana (Anderson \& Domsch, 1996), sendo expresso em $\mathrm{mg} \mathrm{g}^{-1} \mathrm{dia}^{-1} \mathrm{de}$ C$\mathrm{CO}_{2}$ do CBM. Foram estimadas a relação $\mathrm{C}$ da biomassa microbiana/C orgânico (CBM/C) e a relação $\mathrm{N}$ da biomassa microbiana/ $\mathrm{N}$ total $(\mathrm{NBM} / \mathrm{N})$, de acordo com Sparling (1992).

Os dados foram submetidos à análise de variância, segundo em delineamento inteiramente casualizado com quatro repetições nas análises dos atributos químicos e microbianos. Adotou-se o teste $\mathrm{F}$ a $5 \%$. De forma complementar, utilizou-se para comparação 
de médias o teste de Tukey a $5 \%$. Cada cobertura florestal foi considerada um tratamento de efeito fixo, a exemplo do procedimento empregado por Lugo et al. (1990).

Os dados também foram submetidos à análise multivariada por meio de componentes principais, técnicas de agrupamento (método de Tocher) e análise de fatores. A análise de componentes principais e o método de Tocher possibilitam o estudo da similaridade ou dissimilaridade inter e intragrupos e também a avaliação da importância de cada variável sobre a variação total disponível entre as coberturas estudadas (Cruz \& Regazzi, 1994). A análise de fatores permite descrever as relações de covariância entre muitas variáveis com base num pequeno número de quantidades aleatórias, não-observáveis, chamados fatores. As variáveis são agrupadas por meio de suas correlações, ou seja, aquelas pertencentes a um mesmo grupo serão fortemente correlacionadas entre si, mas pouco correlacionadas com as variáveis de outro grupo. Cada grupo representará um fator, responsável pelas correlações observadas (Johnson \& Wichern, 1998).

\section{RESULTADOS E DISCUSSÃO}

\section{Sobrevivência e crescimento das espécies florestais}

As espécies apresentaram sobrevivência no campo acima de $70 \%$ e bom crescimento em altura e diâmetro (DAP). Entre as leguminosas, o sabiá apresentou a maior taxa de sobrevivência, enquanto a acácia mostrou melhor crescimento, especialmente entre os 27 e 44 meses (Quadro 1). A redução dos valores médios do DAP para o sabiá, de 15 para 27 meses, deveu-se à poda das ramificações em excesso na base do tronco, que dificultavam a medição devido à presença de espinhos.

Na acácia e no sabiá, aos 27 meses de idade, houve fechamento do dossel. Isso possibilitou rápida cobertura e proteção do solo à ação erosiva das chuvas.
No eucalipto, contudo, apesar do crescimento similar ao das leguminosas, não houve o fechamento do dossel até a idade de 44 meses (Quadro 1).

\section{Atributos químicos do solo}

A fertilidade do solo sob as coberturas vegetais, de maneira geral, foi baixa, exceto para os teores elevados de K (Quadro 2). Os atributos químicos do solo ( $\mathrm{pH}$, $\mathrm{Al}^{3+}, \mathrm{P}$ e SB), com base na técnica de agrupamento de Tocher, permitiram que as coberturas vegetais fossem reunidas em dois grupos: o primeiro, formado pelo sabiá, pasto, eucalipto e acácia; e o segundo, pela capoeira. A variável que mais contribuiu para a distinção dos grupos foi o $\mathrm{pH}(60 \%)$, seguido da SB (30 \%) e do Al (10\%), sendo nula a contribuição do P. Esses mesmos atributos puderam ser distribuídos em dois componentes principais, explicando 99,17\% da variação total entre as coberturas vegetais $(85,29 \%$ para o primeiro componente e $13,88 \%$ para o segundo). A figura 1 mostra a dispersão gráfica das coberturas vegetais em relação aos dois primeiros componentes principais; observa-se que a acácia apresenta baixa similaridade em relação aos demais indivíduos que compõem o grupo 1. Considerando o solo da capoeira como referência, ocorreu aumento significativo da fertilidade dos solos nas demais coberturas vegetais, principalmente pela redução da acidez e elevação da soma de bases, à exceção dos teores de C orgânico e de $\mathrm{N}$ total (Quadro 2). O P disponível não variou entre as coberturas vegetais (Quadro 2). Esse resultado revela a grande estabilidade do $\mathrm{P}$ em solos muito intemperizados. O P mineralizado, quando não absorvido pelas plantas, passaria rapidamente para formas não-lábeis (Novais \& Smyth, 1999). Nesse sentido, a disponibilidade de $\mathrm{P}$ seria regulada pela fração de $\mathrm{P}$ orgânico. Em solos sob fragmentos de mata atlântica montana, povoamento de $C$. citriodora de 20 anos e pastagens degradadas, em Santa M. Madalena, RJ, o P-orgânico correspondeu a $65 \%$ do P lábil (Cunha, 2002). Gama-Rodrigues et al. (1999) observaram melhoria da fertilidade do solo por plantios florestais em relação a fragmentos de floresta natural nos tabuleiros costeiros do sudeste da Bahia. Por outro

Quadro 1. Sobrevivência, altura total e diâmetro à altura do peito (DAP) de três espécies florestais

\begin{tabular}{|c|c|c|c|c|c|c|c|}
\hline \multirow{2}{*}{ Cobertura } & \multirow{2}{*}{$\begin{array}{c}\text { Sobrevivência } \\
\text { (mês) } \\
15\end{array}$} & \multicolumn{3}{|c|}{ Altura total ${ }^{(1)}$} & \multicolumn{3}{|c|}{$\operatorname{DAP}^{(1)}$ (meses) } \\
\hline & & 15 & 27 & 44 & 15 & 27 & 44 \\
\hline & $\%$ & \multicolumn{3}{|c|}{$-\mathrm{m}$} & \multicolumn{3}{|c|}{$\mathrm{cm}$} \\
\hline Acácia & 74 & $2,24(1,05)$ & $4,66(1,48)$ & $8,40(2,23)$ & $2,87(1,14)$ & $4,23(1,95)$ & $6,94(2,58)$ \\
\hline Sabiá & 87 & $3,27(1,44)$ & $5,23(2,45)$ & $6,15(2,88)$ & $3,57(1,81)$ & $2,79(2,52)$ & $3,94(3,24)$ \\
\hline Eucalipto & 70 & $3,42(0,35)$ & $6,85(0,67)$ & $9,59(0,65)$ & $3,95(1,81)$ & $6,16(2,52)$ & $8,45(3,24)$ \\
\hline
\end{tabular}

(1) Valor entre parênteses indica o desvio-padrão; DAP inferior a 1,0 cm não foi computado. 
Quadro 2. Atributos químicos do Latossolo Vermelho- Amarelo sob diferentes coberturas vegetais

\begin{tabular}{|c|c|c|c|c|c|c|c|c|c|c|c|}
\hline Cobertura & $\mathbf{p H}$ & $\mathrm{Ca}^{2+}$ & $\mathrm{Mg}^{2+}$ & $\mathrm{Al}^{3+}$ & $\mathbf{P}$ & $\mathbf{K}$ & C orgânico & N total & SB & $\mathbf{H}+\mathbf{A l}$ & $\mathrm{CTC}_{\mathbf{p H}} 7,0$ \\
\hline & & 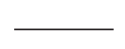 & $\mathrm{cmol}_{\mathrm{c}} \mathrm{dm}^{-3}$ & - & \multicolumn{2}{|c|}{$-\mathrm{mgdm}{ }^{-3}-$} & \multicolumn{2}{|c|}{$\mathrm{g} \mathrm{kg}^{-1}$} & 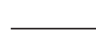 & $\mathrm{cmol}_{\mathrm{c}} \mathrm{dm}^{-3}$ & \\
\hline Acácia & $5,24 \mathrm{a}$ & $1,11 \mathrm{bc}$ & $0,76 \mathrm{bc}$ & $0,33 \mathrm{~d}$ & $2,57 \mathrm{a}$ & $112 \mathrm{~b}$ & $12,28 \mathrm{c}$ & $1,22 \mathrm{~b}$ & $2,16 \mathrm{~b}$ & $6,16 \mathrm{c}$ & $8,32 \mathrm{~b}$ \\
\hline Sabiá & $5,10 \mathrm{~b}$ & $1,45 \mathrm{ab}$ & $0,95 \mathrm{a}$ & $0,57 \mathrm{c}$ & $2,68 \mathrm{a}$ & $104 \mathrm{~b}$ & $11,94 \mathrm{c}$ & $1,41 \mathrm{ab}$ & $2,67 \mathrm{a}$ & $6,39 \mathrm{c}$ & $9,06 \mathrm{ab}$ \\
\hline Eucalipto & $4,89 \mathrm{c}$ & $0,96 \mathrm{~cd}$ & $0,80 \mathrm{~b}$ & $0,83 \mathrm{~b}$ & $2,75 \mathrm{a}$ & $103 \mathrm{~b}$ & $13,24 \mathrm{~b}$ & $1,15 \mathrm{~b}$ & $2,02 \mathrm{~b}$ & $7,45 \mathrm{~b}$ & $9,47 \mathrm{ab}$ \\
\hline Pasto & $5,05 \mathrm{~b}$ & 1,54 a & $0,79 \mathrm{~b}$ & $0,67 \mathrm{bc}$ & $2,73 \mathrm{a}$ & $133 \mathrm{a}$ & $14,99 \mathrm{a}$ & $1,69 \mathrm{a}$ & $2,67 \mathrm{a}$ & $7,57 \mathrm{~b}$ & $10,24 \mathrm{a}$ \\
\hline Capoeira & $4,35 \mathrm{~d}$ & $0,66 \mathrm{~d}$ & $0,64 \mathrm{c}$ & $1,43 \mathrm{a}$ & $2,82 \mathrm{a}$ & $65 \mathrm{c}$ & $13,62 \mathrm{~b}$ & $1,49 \mathrm{a}$ & $1,47 \mathrm{c}$ & $8,72 \mathrm{a}$ & $10,19 \mathrm{a}$ \\
\hline
\end{tabular}

Letras iguais nas colunas não diferem significativamente pelo teste de Tukey a $5 \%$.

lado, o fato de o solo sob pastagem no presente trabalho apresentar maior fertilidade do que a capoeira (Quadro 2) revela que o nível de degradação da pastagem se restringe à disponibilidade de forragem, em decorrência do manejo inadequado de pastejo.

O solo sob eucalipto apresentou $\mathrm{pH}$ inferior aos das leguminosas, mostrando, correspondentemente, maior teor de Al trocável e valor de acidez potencial (Quadro 2). Resultados similares foram observados por Vezzani et al. (2001) em plantios de Eucalyptus saligna e Acacia mearnsii com 45 meses de idade. Entretanto, Franco et al. (1996), avaliando a contribuição de Acacia mangium e Eucalyptus pellita, com oito anos de idade, na recuperação de solos degradados, verificaram valores de $\mathrm{pH}$ do solo sob a leguminosa inferiores aos observados no solo sob eucalipto. Isso seria em decorrência de a acácia, em associação às bactérias diazotróficas, promover maior extrusão de prótons, com conseqüente redução de $\mathrm{pH}$ do solo. Quanto à soma de bases, o solo sob eucalipto

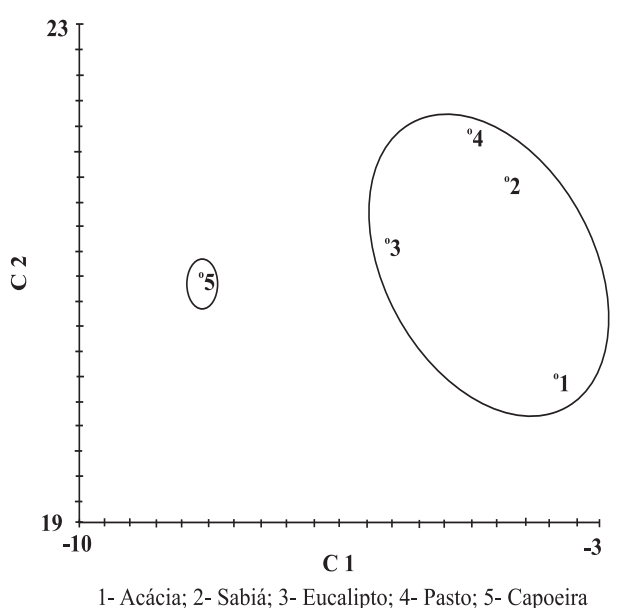

Figura1. Dispersão gráfica das coberturas vegetais em relação aos dois primeiros componentes principais, com base no $\mathrm{pH}, \mathrm{Al}^{3+}$, $\mathrm{P}$ e $\mathrm{SB}$ do solo sob diferentes coberturas vegetais. foi inferior em relação ao sabiá e ao pasto (Quadro 2). Garay et al. (2003) observaram em plantio de Eucalyptus grandis, com sete anos de idade, menores e maiores valores de soma de bases e de $\mathrm{pH}$, respectivamente, em relação ao plantio de Acacia mangium.

Entre os plantios florestais, a acácia e o sabiá mostraram menor teor de C orgânico (Quadro 2). O decréscimo da CTC no solo sob sabiá foi concomitante com a redução do teor de C orgânico, quando comparado ao pasto (Quadro 2). O plantio provavelmente estimulou a rápida decomposição da MO acumulada na pastagem. Contudo, é possível que, com o passar do tempo, quando houver significativo acúmulo de serapilheira, os teores de MO nos solos sob os plantios venham a alcançar ou superar os do pasto e da capoeira.

Plantios de leguminosas florestais fixadoras de $\mathrm{N}_{2}$, na fase de estabilização do estande, normalmente promovem aumentos nos teores de $\mathrm{MO}$ e de $\mathrm{N}$ (total e disponível) no solo e, em alguns casos, decréscimo do $\mathrm{pH}$ e dos teores de $\mathrm{P}$ e de bases trocáveis (Binkley \& Giardina, 1997). Solos sob plantios de espécies de leguminosas da mata atlântica, com idade de 22 anos, apresentaram maiores teores de C orgânico em relação aos fragmentos de floresta natural (Gama-Rodrigues et al., 1999). Cunha (2002) constatou decréscimos dos teores de $\mathrm{C}$ orgânico na conversão de floresta natural em pastagens.

\section{Atributos microbianos do solo}

O teor máximo de CBM (C da biomassa microbiana) na capoeira e o mínimo na acácia apresentou variação de $69,5 \%$. As demais coberturas vegetais apresentaram pequenas variações nos teores de CBM. Os maiores teores médios de NBM ( $\mathrm{N}$ da biomassa microbiana) foram encontrados no pasto, eucalipto e sabiá. A RA (respiração acumulada) também apresentou valor superior no pasto; o eucalipto, atividade microbiana intermediária; e a capoeira e as leguminosas, menores valores (Quadro 3). Os teores de CBM e NBM dos solos sob capoeira, pastagem e eucalipto foram inferiores, e os de RA, semelhantes 
Quadro 3. Atributos microbianos dos solos sob diferentes coberturas vegetais

\begin{tabular}{|c|c|c|c|c|c|c|c|}
\hline Cobertura & $\mathrm{CBM}^{(1)}$ & NBM & $\mathbf{R A}$ & $q \mathrm{CO}_{2}$ & $\mathrm{CBM} / \mathrm{C}$ & NBM/N & $\mathrm{C} / \mathrm{N}$ microbiana \\
\hline & \multicolumn{3}{|c|}{$\mu \mathrm{g} \mathrm{g}^{-1}$ no solo seco } & & \multicolumn{2}{|c|}{$\%$} & \\
\hline Acácia & $241,53 \mathrm{~b}$ & $64,87 \mathrm{~b}$ & $31,36 \mathrm{~b}$ & $18,54 \mathrm{ab}$ & $1,97 \mathrm{a}$ & $5,32 \mathrm{~b}$ & $3,72 \mathrm{~b}$ \\
\hline Sabiá & $325,92 \mathrm{ab}$ & 92,38 a & $26,30 \mathrm{~b}$ & $11,53 \mathrm{~b}$ & $2,73 \mathrm{a}$ & $6,55 \mathrm{ab}$ & $3,53 \mathrm{~b}$ \\
\hline Eucalipto & $257,76 \mathrm{~b}$ & 98,75 a & $45,64 \mathrm{ab}$ & 25,29 a & $1,95 \mathrm{a}$ & $8,59 \mathrm{a}$ & $2,61 \mathrm{~b}$ \\
\hline Pasto & 436,25 a & $104,75 \mathrm{a}$ & 58,87 a & $19,27 \mathrm{a}$ & $2,91 \mathrm{a}$ & $6,21 \mathrm{ab}$ & $4,16 \mathrm{~b}$ \\
\hline Capoeira & 409,30 a & $64,65 \mathrm{~b}$ & $37,52 \mathrm{~b}$ & $13,09 \mathrm{~b}$ & $3,00 \mathrm{a}$ & $4,34 \mathrm{~b}$ & $6,33 \mathrm{a}$ \\
\hline
\end{tabular}

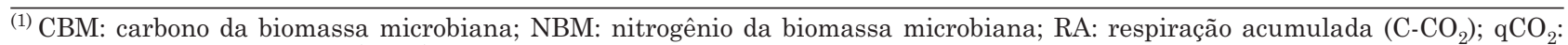
quociente metabólico (mg g ${ }^{-1}$ dia $^{-1}$ de $\mathrm{C}-\mathrm{CO}_{2}$ do $\mathrm{CBM}$ ); $\mathrm{CBM} / \mathrm{C}$ : relação $\mathrm{C}$ microbiano/C orgânico; NBM/N: relação nitrogênio microbiano/ $\mathrm{N}$ total; $\mathrm{C} / \mathrm{N}$ microbiana: relação $\mathrm{C} / \mathrm{N}$ microbiana. Letras iguais nas colunas não diferem significativamente pelo teste de Tukey a $5 \%$.

aos obtidos por Monteiro (2001) em solos cobertos com esse mesmo tipo de vegetação, na região serrana de Santa M. Madalena, RJ. Estudos realizados por Reis et al. (2000) verificaram menor teor de CBM em solo sob eucalipto do que em solo sob vegetação espontânea. Todavia, esses autores não observaram diferenças significativas entre os teores de CBM em solos sob eucalipto, acácia, albízia e consórcio eucalipto-albízia.

O teor de CBM na capoeira pode estar relacionado à melhor qualidade da serapilheira, devido à combinação de diferentes espécies nesse ecossistema (Gama-Rodrigues et al., 1997, 1999), favorecendo a imobilização de $\mathrm{C}$ pela biomassa microbiana. No pasto, o teor de CBM deve-se, provavelmente, à alta taxa de renovação das raízes finas e à entrada de serapilheira mais facilmente decomponível, resultando em C disponível para a população microbiana e aumento da sua biomassa (Geraldes et al., 1995; Fernandes et al., 1997; Feigl et al., 1998; Luizão et al., 1999).

Os atributos químicos (C orgânico e $\mathrm{N}$ total) e os microbianos (CBM, NBM e RA), com base no método de Tocher, permitiram que as diferentes coberturas vegetais fossem distribuídas em dois grupos: o primeiro, formado por acácia, sabiá, eucalipto e capoeira; e o segundo, pelo pasto. Dos atributos microbianos, os que mais contribuíram para a formação dos grupos foram o NBM, com $50 \%$, seguido do CBM com 20 \% e da RA com $10 \%$. O C orgânico apresentou contribuição nula, e o $\mathrm{N}$ total contribuiu com $20 \%$. Esses resultados demonstraram que os atributos microbianos foram mais discriminantes que o teor de $\mathrm{C}$ orgânico no tocante às mudanças na cobertura vegetal. Resultados similares foram reportados por Gama-Rodrigues (1999) e Monteiro (2001). O C orgânico, N total, CBM, NBM e RA foram distribuídos em dois componentes principais, explicando $87,13 \%$ da variação total entre as coberturas vegetais $(60,63 \%$ para o primeiro componente e $26,50 \%$ para o segundo) (Figura 2).

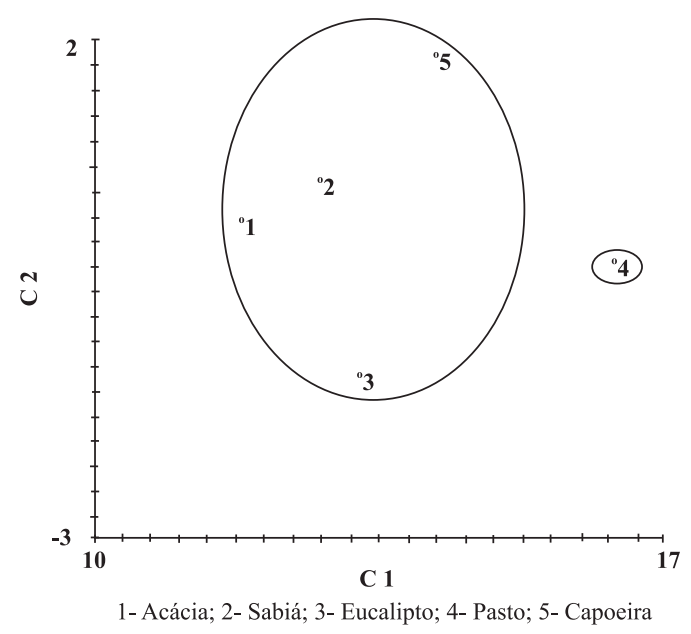

Figura 2. Dispersão gráfica das coberturas vegetais em relação aos dois primeiros componentes principais, com base nos teores de $\mathrm{C}$ orgânico, $\mathrm{N}$ total, CBM, NBM e C-CO $\mathrm{CO}_{2}$ do solo sob diferentes coberturas vegetais.

A análise de fatores permitiu que os atributos químicos e microbianos do solo fossem distribuídos em dois fatores. O primeiro foi formado pelo $\mathrm{pH}, \mathrm{Al}^{3+}$, $\mathrm{P}, \mathrm{SB}$ e C orgânico, caracterizando o papel da MO na disponibilidade de nutrientes e nos componentes da acidez do solo (Quadro 4). Nos solos de avançado intemperismo, a MO constitui-se numa das principais fontes de acidez, juntamente com o $\mathrm{Al}^{3+}$ trocável (Bohnen et al., 2000). Além disso, os menores teores de $\mathrm{Ca}^{2+}, \mathrm{Mg}^{2+}$ e $\mathrm{K}$ no solo sob a capoeira sugerem maior quantidade desses elementos imobilizados na biomassa da parte aérea, acarretando aumento da acidez do solo (Quadro 2).

$\mathrm{O}$ segundo fator foi constituído pelo $\mathrm{N}$ total, CBM, NBM e RA, caracterizando os processos de imobilização e mineralização exercidos pela biomassa microbiana "status microbiano" e a lógica dependência da atividade dos microrganismos do solo pelos níveis de $\mathrm{N}$ no solo 
(Quadro 4). Esses resultados sugeririam que a biomassa microbiana e sua atividade são mais influenciadas pelo teor de $\mathrm{N}$ do que pelo C. Resultados similares foram obtidos por Martens (1987), van der Werf \& Werstrate (1987) e Gama-Rodrigues (1997).

A relação $\mathrm{CBM} / \mathrm{C}$ pouco variou entre as coberturas vegetais; por outro lado, o $q \mathrm{CO}_{2}$ e as relações $\mathrm{NBM} / \mathrm{N}$ e $\mathrm{C} / \mathrm{N}$ microbiana apresentaram diferenças entre o valor máximo e o mínimo de $132,1,110,5$ e 176,5 \%, respectivamente. As médias dos índices microbianos foram menores do que as obtidas por Ndaw (2003) em solos sob eucalipto, pasto e floresta natural no Norte Fluminense. Ao contrário do observado no presente trabalho, Gama-Rodrigues et al. (1997) verificaram valores de $q \mathrm{CO}_{2}$ mais elevados em solos sob capoeira e angico do que em solos sob eucalipto e pinheiro. Esses autores sugeriram que o maior valor $q \mathrm{CO}_{2}$ estaria relacionado à maior taxa de decomposição dessas espécies, em razão da alta qualidade de serapilheira produzida.

Quando se consideraram os índices microbianos do solo ( $\mathrm{qCO}_{2}, \mathrm{CBM} / \mathrm{C}, \mathrm{NBM} / \mathrm{N}$ e $\mathrm{C} / \mathrm{N}$ microbiana), ocorreu a formação de três grupos heterogêneos entre si, pelo método de Tocher: a acácia, o pasto e o sabiá formaram o primeiro grupo; o eucalipto, o segundo; e a capoeira, o terceiro. A variável que mais contribuiu para a distinção dos grupos foi a relação $\mathrm{NBM} / \mathrm{N}$ e o $q \mathrm{CO}_{2}$, ambos com $30 \%$, seguidos das relações $\mathrm{CBM} / \mathrm{C}$ e C/N microbiana, cada uma contribuindo com $20 \%$. Esses resultados demonstraram que os índices microbianos foram mais discriminantes do que os atributos químicos e microbianos. Desse modo, os índices microbianos permitiriam um entendimento mais detalhado da eficiência da biomassa microbiana nos processos de imobilização e mineralização de $\mathrm{C}$ e $\mathrm{N}$, devido à inter-relação entre os atributos químicos,

Quadro 4. Cargas fatoriais, após a rotação pelo método varimax, na análise fatorial da matriz de correlações simples de nove variáveis associadas aos atributos do solo sob diferentes coberturas vegetais

\begin{tabular}{lrl}
\hline Atributo do solo & Fator I & Fator II \\
\hline $\mathrm{pH}$ & 0,993 & 0,352 \\
$\mathrm{Al}^{3+}$ & $-0,976$ & $-0,290$ \\
$\mathrm{P}$ & $-0,807$ & 0,460 \\
$\mathrm{SB}$ & 0,830 & 0,420 \\
C orgânico & $-0,691$ & 0,508 \\
N total & 0,430 & 0,721 \\
CBM & $-0,285$ & 0,873 \\
NBM & $-0,220$ & 0,772 \\
RA & $-0,043$ & 0,857 \\
\hline
\end{tabular}

CBM: carbono da biomassa microbiana do solo; NBM: nitrogênio da biomassa microbiana do solo; RA: respiração acumulada do solo. que definem a qualidade e quantidade da MO (C orgânico e N total), e os atributos microbianos do solo (CBM, NBM e RA) (Gama-Rodrigues, 1999). As relações CBM/C orgânico e NBM/N total expressam a eficiência da população de microrganismos em imobilizar C e N (Wardle, 1992). Além disso, indicam o quanto a biomassa microbiana representa de reservatório "lábil" na dinâmica desses elementos. Já a relação $\mathrm{C} / \mathrm{N}$ microbiana indica o potencial de mineralização de $\mathrm{N}$ e mudanças na composição microbiana, em que valores acima de 10 indicariam predomínio de fungos e, abaixo deste valor, predomínio de bactérias (Campbell et al., 1991; Li et al., 2004).

Os índices microbianos foram distribuídos em dois componentes principais, explicando 91,24\% da variação total entre as coberturas vegetais $(76,81 \%$ para o primeiro componente e $14,44 \%$ para o segundo). A figura 3 mostra a dispersão gráfica das coberturas vegetais em relação aos dois primeiros componentes principais, confirma os grupos formados pelo método de Tocher e demonstra a baixa similaridade do sabiá em relação aos demais indivíduos do grupo 1.

A análise de fatores permitiu que os índices microbianos do solo fossem distribuídos em dois fatores. O primeiro, formado pelas relações CBM/C e $\mathrm{NBM} / \mathrm{N}$, estaria refletindo a qualidade da $\mathrm{MO}$ e a disponibilidade de nutrientes (Quadro 5). O segundo, formado pela relação $\mathrm{C} / \mathrm{N}$ microbiana e $q \mathrm{CO}_{2}$, caracterizaria a relação entre as condições de qualidade e de estresse da MO e a disponibilidade de N (Quadro 5). $\mathrm{O} q \mathrm{CO}_{2}$ é considerado um índice metabólico para avaliar o efeito das condições de estresse sobre a atividade da biomassa microbiana (Anderson \& Domsch, 1993). Já as relações C/N microbiana, CBM/ $\mathrm{C}$ e NBM/N expressam a eficiência da biomassa microbiana em imobilizar $\mathrm{C}$ e $\mathrm{N}$ em função da qualidade

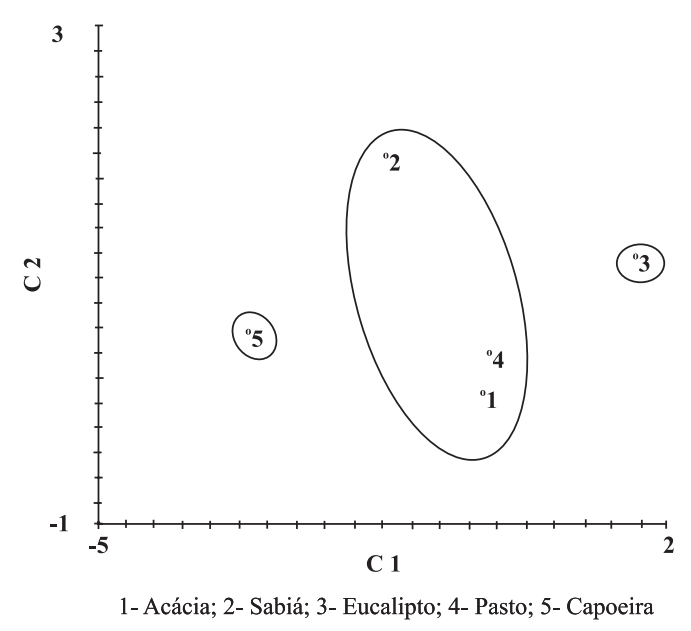

Figura 3. Dispersão gráfica das coberturas vegetais em relação aos dois primeiros componentes principais, com base no $q \mathrm{CO}_{2}$ e nas relações $\mathrm{CBM} / \mathrm{C}$, NBM/N e C/N microbiana do solo sob diferentes coberturas vegetais. 
nutricional da MO (Joergensen \& Brookes, 1990; Sparling, 1992).

Quadro 5. Cargas fatoriais, após a rotação pelo método varimax, na análise fatorial da matriz de correlações simples de quatro variáveis associadas aos índices microbianos do solo sob diferentes coberturas vegetais

\begin{tabular}{crc}
\hline $\begin{array}{c}\text { Índice } \\
\text { microbianos do solo }\end{array}$ & Fator I & Fator II \\
\hline $\mathrm{qCO}_{2}$ & $-0,433$ & $\mathbf{0 , 8 3 0}$ \\
$\mathrm{CBM} / \mathrm{C}$ & $\mathbf{0 , 9 0 2}$ & $-0,343$ \\
$\mathrm{NBM} / \mathrm{N}$ & $\mathbf{- 0 , 8 9 5}$ & 0,353 \\
$\mathrm{C} / \mathrm{N}$ microbiana & 0,286 & $\mathbf{0 , 9 1 4}$
\end{tabular}

(1) $\mathrm{CCO}_{2}$ : quociente metabólico do solo; $\mathrm{CBM} / \mathrm{C}$ : relação $\mathrm{C}$ microbiano/C orgânico do solo; NBM/NS - relação N microbiano/ $\mathrm{N}$ total do solo; $\mathrm{C} / \mathrm{N}$ microbiana: relação $\mathrm{C} / \mathrm{N}$ microbiana do solo.

\section{CONCLUSÕES}

1. As espécies florestais, na fase inicial de crescimento, revelaram-se promissoras em melhorar a fertilidade do solo, em relação à capoeira, no que tange ao $\mathrm{pH}$ e à soma de bases, apesar da redução dos teores de MO.

2. A pastagem mostrou potencial de manter o teor de $\mathrm{MO}$ e melhorar a fertilidade do solo, em relação à capoeira.

3. Os teores de $\mathrm{C}$ e $\mathrm{N}$ microbiano, pelo método de Tocher, foram mais discriminantes que o teor de $\mathrm{C}$ orgânico do solo às mudanças na cobertura vegetal.

4. Os índices microbianos $\left(q \mathrm{CO}_{2}, \mathrm{CBM} / \mathrm{C}, \mathrm{NBM} / \mathrm{N}\right.$ e $\mathrm{C} / \mathrm{N}$ microbiana), pelo método de Tocher, foram mais discriminantes que os atributos químicos (C orgânico e $\mathrm{N}$ total) e microbianos (CBM, NBM e RA) em aferir a dissimilaridade entre as coberturas vegetais.

\section{AGRADECIMENTO}

À Fundação de Amparo à Pesquisa do Estado do Rio de Janeiro (FAPERJ), pelo auxílio financeiro, e ao Instituto de Pesquisa Pró-Natura, pelo apoio logístico nos trabalhos de campo. Ao Prof. Cosme Damião Cruz, pelo auxílio na análise multivariada.

\section{LITERATURA CITADA}

ANDERSON, J.D. \& INGRAM, J.S.I. Tropical soil biology and fertility: A handbook of methods. 2.ed. Wallingford, $\mathrm{CAB}$ International, 1993. 171p.
ANDERSON, J.P.E. \& DOMSCH, K.H. The metabolic quotient for $\mathrm{CO}_{2}\left(\mathrm{qCO}_{2}\right)$ as a specific activity parameter to assess the effects of environmental conditions, such as $\mathrm{pH}$, on the microbial biomass of forest soils. Soil Biol. Biochem., 25:393-395, 1996.

ARSHAD, M. A. \& MARTIN, S. Identifying critical limits for soil quality indicators in agro- ecosystems. Agric., Ecosyst. Environ., 88:153-160, 2002.

BARROS, N.F. \& COMERFORD, N.B. Sustentabilidade da produção de florestas plantadas na região tropical. In: ALVAREZ V., V.H.; SCHAEFER, C.E.G.R.; BARROS, N.F.; MELLO, J.W.V. \& COSTA, L.M., eds. Tópicos em ciência do solo. Viçosa, MG, Sociedade Brasileira de Ciência do Solo, 2002. p.487-592.

BARTLETT, R.J. \& ROSS, D.S. Colorimetric determination of oxidizable carbon in acid soil solutions. Soil Sci. Soc. Am. J. 52:1191-1192, 1988.

BINKLEY, D. \& GIARDINA, C. Nitrogen fixation in tropical forest plantations. In: NAMBIAR, E.K.S. \& BROWN, A.G., eds. Management of soil, nutrients and water in tropical plantation forests. Camberra, ACIAR, 1997. p.297337.

BOHNEN, H.; MEURER, E.J. \& BISSANI, C.A. Solos ácidos e solos afetados por sais. In: MEURER, E.J., ed. Fundamentos de química do solo. Porto Alegre, Genesis, 2000. p.109-122.

BOUMA, J. Land quality indicators of sustainable land management across scales. Agric., Ecosyst. Environ., 88:129-136, 2002.

CAMPBELL, C.A.; BIEDERBECK, V.O.; ZENTNER, R.P. \& LAFOND, G.P. Effect of crop rotations and cultural practices on soil organic matter, microbiol biomass and respiration in a thin black Chernozen. Can. J. Soil Sci., 71:363-376, 1991.

CAMPELLO, E.F.C. Sucessão vegetal na recuperação de áreas degradadas. In: DIAS, L.E. \& GRIFFITH, J.J., eds. Recuperação de áreas degradadas. Viçosa, MG, Universidade Federal de Viçosa, 1998. p.183-196.

CAMPELlO, E.F.C.; FRANCO, A.A. \& DIAS, L.E. Monitoramento ambiental. R. Ação Amb., 10:24-25, 2000.

CUNHA, G.M. Ciclagem de nutrientes em florestas montanas e em Eucalyptus citriodora na região norte fluminense. Campos dos Goytacazes, Universidade Estadual do Norte Fluminense, 2002. 122p.(Tese de Doutorado)

CRUZ, C.D. \& REGAZZI, A.J. Modelos biométricos aplicados ao melhoramento genético. Viçosa, MG, Universidade Federal de Viçosa, 1994. 390p.

DORAN, J.W. \& ZEISS, M.R. Soil health and sustainability: Managing the biotic component of soil quality. Appl. Soil Ecol., 15:3-11, 2000.

EMPRESA BRASILEIRA DE PESQUISA AGROPECUÁRIA EMBRAPA. Centro Nacional de Pesquisa de Solos. Manual de métodos de análise de solo. 2.ed. Rio de Janeiro, 1997. 212p. 
FEIGL, B.J.; CERRI, C.C. \& BERNOUX, M. Balanço de carbono e biomassa microbiana em solos da Amazônia. In: MELO, I.S. \& AZEVEDO, J.L., eds. Ecologia microbiana. Jaguariúna, Embrapa, 1998. 486p.

FERNANDES, S.A.P.; FEIGL, B.J. \& CERRI, C.C. Ação de fertilizante fosfatado na biomassa microbiana de solos sob pastagem e capoeira em Rondônia. In: CONGRESSO BRASILEIRO DE CIÊNCIA DO SOLO, 26., Rio de Janeiro, 1997. Anais. Rio de Janeiro, 1997. CD-ROM

FRANCO, A.A.; CAMPELLO, E.F.C.; DIAS, L.E. \& FARIA, S.M. Uso de leguminosas associadas a microrganismos na revegetação de áreas de mineração de bauxita em Porto Trombetas - PA. Itaguaí, Embrapa Agrobiologia, 1996. 69p. (Documentos, 27)

FRANCO, A.A. Fixação biológica do nitrogênio na agricultura tropical. In: ALVAREZ V., V.H.; FONTES, L.E. \& FONTES, M.P., eds. O solo nos grandes domínios morfoclimáticos do Brasil e o desenvolvimento sustentado. Viçosa, MG, Sociedade Brasileira de Ciência do Solo, 1996. p.505-523.

FRANCO, A.A. \& FARIA, S.M. The contribution of $\mathrm{N}_{2}$-fixing tree legumes to land reclamation and sustainability in the tropics. Soil Biol. Biochem., 29:897-903, 1997.

GAMA-RODRIGUES, E.F. Carbono e nitrogênio da biomassa microbiana do solo e da serapilheira de povoamentos de eucalipto. Seropédica, Universidade Federal Rural do Rio de Janeiro, 1997. 108p. (Tese de Doutorado)

GAMA-RODRIGUES, E.F. Biomassa microbiana e ciclagem de nutrientes. In: SANTOS, G.A. \& CAMARGO, F.A.O., eds. Fundamentos da matéria orgânica do solo Ecossistemas tropicais e subtropicais. Porto Alegre, Genesis, 1999. p.227-244.

GAMA-RODRIGUES, E.F.; GAMA-RODRIGUES, A.C. \& BARROS, N.F. Biomassa microbiana de carbono e de nitrogênio de solos sob diferentes coberturas florestais. R. Bras. Ci. Solo, 21:361-365, 1997.

GAMA-RODRIGUES, A.C.; BARROS, N.F. \& MENDONÇA, E.S. Alterações edáficas sob plantios puros e misto de espécies florestais nativas do Sudeste da Bahia, Brasil. R. Bras. Ci. Solo, 23:581-592, 1999.

GAMA-RODRIGUES, A.C. \& MAY, P. SAF e o planejamento do uso da terra: Experiência na região Norte Fluminense-RJ. In: MACÊDO, J.L.V.; WANDELLI, E.V. \& SILVA J., J.P., eds. Sistemas agroflorestais: Manejando a biodiversidade e compondo a paisagem rural; CONGRESSO BRASILEIRO DE SISTEMAS AGROFLORESTAIS, 3., Manaus, 2001. Anais. Manaus, Embrapa Amazônia Ocidental, 2001. p.130-145.

GARAY, I.; KINDEL, A.; CARNEIRO, R.; FRANCO, A.A.; BARROS, E. \& ABBADIE, L. Comparação da matéria orgânica e de outros atributos do solo entre plantações de Acacia mangium e Eucalyptus grandis. R. Bras. Ci. Solo, 27:705-712, 2003.
GERALDES, P.A.; CERRI, C.C. \& FEIGL, B.J. Biomassa microbiana de solo sob pastagens na Amazônia. R. Bras. Ci. Solo, 1:55-60, 1995.

GRISI, B.M. Biomassa e a atividade de microrganismos do solo: Revisão metodológica. R. Nordestina Biol., 10:1-22, 1995.

JOERGENSEN, R.G. \& BROOKES, P.C. Ninhydrin-reactive nitrogen measurements of microbial biomass in $0,5 \mathrm{M} \mathrm{K}_{2} \mathrm{SO}_{4}$ soil extracts. Soil Biol. Biochem., 22:1023-1027, 1990.

JOHNSON, R.A. \& WICHERN, D.W. Applied multivariate statistical analysis. New Jersey, Prentice-Hall, 1998. 816p.

LÁNG, I. The ecological foundations of sustainable land use: Hungarian agriculture and the way to sustainability. In: GREENLAND, D.J. \& SZABOLCS, I., eds. Soil resilience and sustainable land use. Wallingford, CAB International, 1994. p.3-19.

LI, Q.; ALLEN, H.L. \& WOLLUM II, A.G. Microbial biomass and bacterial functional diversity in forest soils: Effects of organic matter removal, compaction, and vegetation control. Soil Biol. Biochem., 36:571-579,2004.

LUGO, A.E.; CUEVAS, E. \& SANCHEZ, M.J. Nutrients and mass in litter and soil of ten tropical tree plantations. Plant Soil, 125:263-280, 1990.

LUIZÃO, R.C.C.; COSTA, E. S. \& LUIZÃO, F.J. Mudanças na biomassa microbiana e nas transformações de nitrogênio do solo em uma seqüência de idades de pastagens após derruba e queima de floresta na Amazônia Central. Acta Amaz., 29:43-56, 1999.

MARTENS, R. Estimation of microbial biomass in soils by the respiration method: Importance of soil $\mathrm{pH}$ and flushing methods for measurement of respired $\mathrm{CO}_{2}$. Soil Biol. Biochem., 19:77-81, 1987.

MONTEIRO, M.T. Carbono, nitrogênio e atividade da biomassa microbiana: Indicadores da qualidade do solo e da serapilheira em sítios florestais do Norte Fluminense. Campos dos Goytacazes, Universidade Estadual do Norte Fluminense, 2001. 74p. (Tese de Mestrado)

MELE, P.M. \& CARTER, M.R. Effect of climatic factors on the use of microbial biomass as an indicator of changes in soil organic matter. In: MULONGOY, K. \& MERCKX, R. Soil organic matter dynamics and sustainability of tropical agriculture. New York, Jonh Wiley \& Sons, 1993. p.57-64.

NDAW, S.M. Diversidade, biomassa e atividade microbiana como indicadores da qualidade do solo e da serapilheira, em sítios sob diferentes coberturas vegetais na região norte fluminense. Campos dos Goytacazes, Universidade Estadual do Norte Fluminense, 2003. 89p. (Tese de Mestrado)

NOVAIS, R.F. \& SMYTH, T.J. Fósforo em solo e planta em condições tropicais. Viçosa, MG, Universidade Federal de Viçosa, 1999. 399p.

REINERT, D.J. Recuperação de solos em sistemas agropastoris. In: DIAS, L.E. \& GRIFFITH, J.J., eds. Recuperação de áreas degradadas. Viçosa, MG, Universidade Federal de Viçosa, 1998. p.163-176. 
REIS, L.L.; CAMPELLO, E.F.C. \& FRANCO, A.A. Atividade microbiana de um Planossolo sob plantio de leguminosas arbóreas utilizadas na recuperação de áreas degradadas em comparação ao eucalipto e vegetação espontânea. In: SIMPÓSIO NACIONAL DE RECUPERAÇÃO DE ÁREAS DEGRADADAS, 4., Blumenau, 2000. Anais. Blumenau, 2000. CD-ROM.

SPARLING, G.P. Ratio of microbial biomass carbon to soil organic carbon as a sensitive indicator of changes in soil organic matter. Aust. J. Soil Res., 30:195-207, 1992.

van de WERF, H. \& VERSTRAETE, W. Estimation of active soil microbial biomass by mathematical analysis of respiration curves: Calibration of test procedures. Soil Biol. Biochem., 19:261-265, 1987.
VANCE, E.D.; BROOKES, P.C. \& JENKINSON, D.S. An extraction method for measuring soil microbial biomass C. Soil Biol. Biochem., 19:703-707, 1987.

VEZZANI, F.M.; TEDESCO, M.J. \& BARROS, N.F. Alterações dos nutrientes no solo e nas plantas em consórcio de eucalipto e acácia negra. R. Bras. Ci. Solo, 25:225-231, 2001.

WARDLE, D.A. A comparative assessment of factors which influence microbial biomass carbon and nitrogen levels in soil. Biol. Rev., 67:321-358, 1992.

YOUNG, A. Agroforestry for soil conservation. Wallingford, CAB International/ICRAF, 1989. 276p. 